\title{
COERCIVENESS IN THE NEUMANN PROBLEM
}

\author{
W. J. SWEENEY
}

Let $M^{\prime}$ be a Riemannian manifold of dimension $n$, let $E, F$, and $G$ be Hermitian vector bundles over $M^{\prime}$, and let

$$
E \stackrel{A}{\longrightarrow} F \stackrel{B}{\longrightarrow} G
$$

be a complex of first order linear partial differential operators. For each complex cotangent vector $\zeta \in T_{x}^{*}\left(M^{\prime}\right) \otimes C$ let

$$
E_{x} \stackrel{a(\zeta)}{\longrightarrow} F_{x} \stackrel{b(\zeta)}{\longrightarrow} G_{x}
$$

denote the sequence of symbol maps corresponding to (1). We shall assume that (2) is exact for all real $\zeta \neq 0$ so that (1) is an elliptic complex.

Now let $M$ be a compact $n$-dimensional manifold-with-boundary which is smoothly imbedded in $M^{\prime}$, and let $r$ be a real $C^{\infty}$ function on $M^{\prime}$ such that $M=\left\{x \in M^{\prime} \mid r(x) \geq 0\right\}$ and $\partial M=\left\{x \in M^{\prime} \mid r(x)=0\right\}$ and such that $d r$ never vanishes on $\partial M$. Our purpose here is to discuss the coercive estimate

$$
\|u\|_{1} \leq c\left\{\left\|A^{*} u\right\|+\|B u\|+\|u\|\right\}
$$

for $u \in C^{\infty}(M, F)$ satisfying the boundary condition

$$
a(d r)^{*} u=0 \text { on } \partial M
$$

where $\|\cdot\|$ denotes the $L_{2}$ norm for sections defined over $M$, and $\|\cdot\|_{1}$ denotes any of the equivalent norms on the Sobolev space $\mathscr{H}_{1}(M, F)$. We prove (Theorem 3) that if the coercive estimate holds for all compact $M \subset M^{\prime}$, and (1) is part of a Spencer complex, then (1) is locally exact; we also show (Theorem 4) that if (2) is exact for all complex $\zeta \neq 0$, then the coercive estimate (3) holds for all compact $M$. Under additional assumptions (Theorem 5) we give explicit necessary and sufficient conditions for (3).

Some of our results here (Theorems 1 and 5, in particular) were suggested by recent work of V. W. Guillemin and S. Sternberg [4]. The author is also indebted to J. J. Kohn for several helpful conversations.

Received May 20, 1971. Supported by Air Force Office of Scientific Research Project AFOSR 68-1467. 


\section{A necessary and sufficient condition}

In this section we obtain a condition which is necessary and sufficient for the estimate

$$
\|u\|_{1} \leq c\left\{\left\|A^{*} u\right\|+\|B u\|+\|u\|\right\}
$$

to hold for all $u \in C^{\infty}(M, F)$ satisfying

$$
a(d r)^{*} u=0 \text { on } \partial M
$$

This condition will not depend on the Riemannian structure on $M^{\prime}$ nor on the Hermitian structures on $E, F, G$; it will be used to obtain the local exactness result in the next section. Some of the methods used here (namely, those used to prove Propositions 3 and 4) are fairly well known (see [2], for example); we give proofs for the sake of completeness. In what follows $c$ will denote a generic constant which is not necessarily the same at each occurrence.

Proposition 1. The estimate (3) holds for all $u \in C^{\infty}(M, F)$ satisfying (4) if and only if there exists a constant $c$ such that

$$
\|u\|_{1} \leq c\left\{\left\|A^{*} u\right\|+\|B u\|+\|u\|+{ }^{\partial}\left\|a(d r)^{*} u\right\|_{1 / 2}\right\}
$$

holds for all $u \in C^{\infty}(M, F)$, where ${ }^{\partial}\|\cdot\|_{1 / 2}$ denotes a Sobolev norm of order $\frac{1}{2}$ on $\partial M$.

Proof. Since the sequence (2) is always exact for real $\zeta \neq 0$, it follows that

$$
G_{x} \stackrel{b(d r)^{*}}{\longrightarrow} F_{x} \stackrel{a(d r)^{*}}{\longrightarrow} E_{x}
$$

is exact for each $x \in \partial M$. Now the dimension of $b(d r)^{*}\left(G_{x}\right)$ is automtically a lower semi-continuous function of $x \in \partial M$; and here, since image $b(d r)^{*}=$ $\operatorname{ker} a(d r)^{*}$, it is also upper semi-continuous. It follows that $\operatorname{ker} a(d r)^{*}=$ image $b(d r)^{*}$ has constant dimension on $\partial M$, and hence $a(d r)^{*}$ has constant rank. Accordingly, there exists a bundle mapping $N: E|\partial M \rightarrow F| \partial M$ such that $a(d r)^{*} \cdot N$ is the identity on the image of $a(d r)^{*}$.

Now let $u \in C^{\infty}(M, F)$ and let $u_{0}$ denote the restriction of $u$ to $\partial M$. According to Theorem 2.5.7 in [6] there exists $v \in C^{\infty}(M, F)$ such that $v=N \cdot a(d r)^{*} u_{0}$ on $\partial M$ such that

$$
\|v\|_{1} \leq c^{\partial}\left\|N \cdot a(d r)^{*} u_{0}\right\|_{1 / 2} \leq c^{\partial}\left\|a(d r)^{*} u\right\|_{1 / 2}
$$

holds with a constant $c$, not depending on $u$. Hence, if (3) holds for all sections satisfying (4), then since $a(d r) *(u-v)=a(d r)^{*} u_{0}-a(d r)^{*} N a(d r)^{*} u_{0}=0$ on $\partial M$, we can obtain (5) by estimating: 


$$
\begin{aligned}
\|u\|_{1} \leq\|u-v\|_{1}+\|v\|_{1} & \leq c\left\{\left\|A^{*}(u-v)\right\|+\|B(u-v)\|+\|u-v\|+\|v\|_{1}\right\} \\
& \leq c\left\{\left\|A^{*} u\right\|+\|B u\|+\|u\|+\|v\|_{1}\right\} \\
& \leq c\left\{\left\|A^{*} u\right\|+\|B u\|+\|u\|+{ }^{o}\left\|a(d r)^{*} u\right\|_{1 / 2}\right\}
\end{aligned}
$$

Since the other half of the proposition is trivial, the proof is complete.

Proposition 2. The estimate (5) holds for all $u \in C^{\infty}(M, F)$ if and only if each point in $M$ has a neighborhood $U$ such that (5) holds for all $u \in C_{0}^{\infty}(M \cap U, F)$.

Proof. Since the symbol sequence (2) is exact for all real $\zeta \neq 0$, the operator $u \mapsto\left(A^{*} u, B u\right)$ is elliptic, and hence (3) holds for all $u$ having compact support in the interior of $M$. In particular, every interior point of $M$ has a neighborhood $U$ such that (5) holds for all $u \in C_{0}^{\infty}(U \cap M, F)$. If every boundary point also has such a neighborhood, then we can cover $M$ with a finite number of these neighborhoods and use a subordinate partition of unity $\left\{\varphi_{i}\right\}$ to obtain

$$
\begin{aligned}
\|u\|_{1}=\left\|\Sigma \varphi_{i} u\right\|_{1} & \leq \Sigma\left\|\varphi_{i} u\right\|_{1} \\
& \leq c \Sigma\left\{\left\|A^{*}\left(\varphi_{i} u\right)\right\|+\left\|B\left(\varphi_{i} u\right)\right\|+\left\|\varphi_{i} u\right\|+{ }^{\partial}\left\|a(d r)^{*}\left(\varphi_{i} u\right)\right\|_{1 / 2}\right\} \\
& \leq c\left\{\left\|A^{*} u\right\|+\|B u\|+\|u\|+{ }^{\partial}\left\|a(d r)^{*} u\right\|_{1 / 2}\right\} \\
& +c \Sigma\left\{\left\|\left[\varphi_{i}, A^{*}\right] u\right\|+\left\|\left[\varphi_{i}, B\right] u\right\|\right\} .
\end{aligned}
$$

Since the commutator terms satisfy $\left\|\left[\varphi_{i}, A^{*}\right] u\right\| \leq c\|u\|$ and $\left\|\left[\varphi_{i}, B\right] u\right\| \leq c\|u\|$, the estimate (5) follows immediately.

In view of Proposition 2 it suffices to work on a neighborhood $U$ of an arbitrary boundary point $x_{0}$. Since $U$ can be taken as a coordinate neighborhood in $M^{\prime}$, we may assume that $U \subset R^{n}$, that $x_{0}=0$, that $x^{n} \geq 0$ on $U \cap M$, and that $x^{n}=0$ on $U \cap \partial M$. In addition, we can always arrange that $d r=d x^{n}$ along $U \cap \partial M$ and that $d x^{1}, \cdots, d x^{n-1}$ span $T_{0}^{*}(\partial M)$, which is considered as a subspace of $T_{0}^{*}(M)$ by means of the Riemannian metric. Finally, we may assume that the bundles $E, F$, and $G$ are trivial over $U$. Thus in the case of $F$, for example, we may choose $f_{1}, \cdots, f_{k} \in C^{\infty}(U, F)$ such that $f_{1}(x), \cdots, f_{k}(x)$ form a basis of $F_{x}$ for each $x \in U$ and such that if $u, v \in C_{0}^{\infty}(U, F)$ are written in the form

$$
u=\Sigma u^{i} f_{i}, \quad v=\Sigma v^{i} f_{i}
$$

with $C^{\infty}$ complex-valued functions $u^{i}, v^{i}$, then the $L_{2}$ inner product is given by

$$
\langle u, v\rangle=\int \sum_{i} u^{i}(x) \overline{v^{i}(x)} d x
$$

We shall call such a system $\left\{f_{1}, f_{2}, \cdots\right\}$ a special frame for $F$ over $U$, and we shall also use special frames $\left\{e_{1}, e_{2}, \ldots\right\}$ for $E$ and $\left\{g_{1}, g_{2}, \ldots\right\}$ for $G$ over $U$. 
The local coordinate $x$ determines a local coordinate $(x, \zeta)=\left(x^{1}, \cdots, x^{n}, \zeta_{1}\right.$, $\left.\cdots, \zeta_{n}\right)$ on $T^{*}\left(M^{\prime}\right) \otimes C$ by means of

$$
U \times C^{n} \ni(x, \zeta) \rightarrow \sum \zeta_{i} d x^{i} \in T_{x}^{*}\left(M^{\prime}\right) \otimes C ;
$$

if we use this coordinate and the special frames just chosen, then the symbols of $A$ and $B$ become matrix valued functions $a(x, \zeta)$ and $b(x, \zeta)$, respectively, for $(x, \zeta) \in U \times C^{n}$. Because of the special character of the frames, the symbol of $A^{*}$ is just the adjoint matrix $a(x, \bar{\zeta})^{*}$.

Now let $\boldsymbol{R}_{+}=\{t \in \boldsymbol{R} \mid t \geq 0\}$ and $D_{t}=-\sqrt{-1} \partial / \partial t$. Then for each $\zeta^{\prime}=$ $\left(\zeta_{1}, \cdots, \zeta_{n-1}\right) \in C^{n-1}$ we have differential operators

$$
\begin{aligned}
& a\left(0, \zeta^{\prime}, D_{t}\right): C^{\infty}\left(\boldsymbol{R}_{+}, F_{0}\right) \rightarrow C^{\infty}\left(\boldsymbol{R}_{+}, F_{0}\right), \\
& b\left(0, \zeta^{\prime}, D_{t}\right): C^{\infty}\left(\boldsymbol{R}_{+}, F_{0}\right) \rightarrow C^{\infty}\left(\boldsymbol{R}_{+}, G_{0}\right) .
\end{aligned}
$$

We have

Proposition 3. There exists a neighborhood $U$ of 0 such that (5) holds for all $u \in C_{0}^{\infty}(M \cap U, F)$ if and only if there exists a constant $C$ such that for every $\zeta^{\prime} \in \boldsymbol{R}^{n-1}$ of unit length the estimate

$$
\begin{aligned}
& \int_{0}^{\infty}|w(t)|^{2} d t+\int_{0}^{\infty}\left|D_{t} w(t)\right|^{2} d t \\
&+C\left\{\int_{0}^{\infty}\left|a\left(0, \zeta^{\prime}, D_{t}\right) * w(t)\right|^{2} d t+\int_{0}^{\infty}\left|b\left(0, \zeta^{\prime}, D_{t}\right) w(t)\right|^{2} d t\right. \\
&\left.+\left|a(0,0,1)^{*} w(0)\right|^{2}\right\}
\end{aligned}
$$

holds for all $w \in C_{0}^{\infty}\left(\boldsymbol{R}_{+}, F_{0}\right)$.

Proof. For $1 \leq i \leq n$ let $D_{i}$ denote $-\sqrt{-1} \partial / \partial x^{i}$. Let $\left\{f_{1}, \cdots, f_{k}\right\}$ be the special frame for $F$ chosen above, and let $D_{i}$ operate on sections of $F$ by $D_{i} u=\sum\left(D_{i} u^{j}\right) f_{j}$ if $u=\sum u^{j} f_{j} \in C_{0}^{\infty}(U, F)$. Then, with $D=\left(D_{1}, \cdots, D_{n}\right)$, we obtain differential operators $a(0, D)^{*}$ and $b(0, D)$ such that

$$
\begin{gathered}
\left\|A^{*} u-a(0, D) * u\right\| \leq \epsilon\|u\|_{1}+c\|u\|, \\
\|B u-b(0, D) u\| \leq \epsilon\|u\|_{1}+c\|u\|, \\
{ }^{\partial}\left\|a(d r)^{*} u-a(0,0,1)^{*} u\right\|_{1 / 2} \leq \epsilon^{\partial}\|u\|_{1 / 2}
\end{gathered}
$$

hold for all $u \in C_{0}^{\infty}(M \cap U, F)$, and the constant $\in$ can be made arbitrarily small by choosing $U$ small enough. In view of the estimate

$$
{ }^{o}\|u\|_{1 / 2} \leq c\|u\|_{1},
$$

(see Theorem 2.5.6 in [6]) we also have 


$$
{ }^{\partial}\left\|a(d r)^{*} u-a(0,0,1)^{*} u\right\|_{1 / 2} \leq \epsilon\|u\|_{1},
$$

where, again, $\epsilon$ can be made arbitrarily small by choosing $U$ small enough. For $U$ sufficiently small it now follows from (9), (10), and (11) that (5) is equivalent to the estimate

$$
\|u\|_{1}^{2} \leq c\left\{\left\|a(0, D)^{*} u\right\|^{2}+\|b(0, D) u\|^{2}+\|u\|^{2}+{ }^{\partial}\left\|a(0,0,1)^{*} u\right\|_{1 / 2}^{2}\right\}
$$

which, after taking Fourier transform in the variable $x^{\prime}=\left(x^{1}, \cdots, x^{n-1}\right)$, becomes

$$
\begin{aligned}
& \int_{\boldsymbol{R}^{n-1}}\left\{\int_{0}^{\infty}\left(1+\left|\xi^{\prime}\right|^{2}\right)\left|\tilde{u}\left(\xi^{\prime}, x^{n}\right)\right|^{2} d x^{n}+\int_{0}^{\infty}\left|D_{n} \tilde{u}\left(\xi^{\prime}, x^{n}\right)\right|^{2} d x^{n}\right\} d \xi^{\prime} \\
& \leq c \int_{\boldsymbol{R}^{n-1}}\left\{\int_{0}^{\infty}\left|a\left(0, \xi^{\prime}, D_{n}\right) * \tilde{u}\left(\xi^{\prime}, x^{n}\right)\right|^{2} d x^{n}\right. \\
& \quad+\int_{0}^{\infty}\left|b\left(0, \xi^{\prime}, D_{n}\right) \tilde{u}\left(\xi^{\prime}, x^{n}\right)\right|^{2} d x^{n}+\int_{0}^{\infty}\left|\tilde{u}\left(\xi^{\prime}, x^{n}\right)\right|^{2} d x^{n} \\
& \left.+\left(1+\left|\xi^{\prime}\right|^{2}\right)^{1 / 2}\left|a(0,0,1)^{*} \tilde{u}\left(\xi^{\prime}, 0\right)\right|^{2}\right\} d \xi^{\prime}
\end{aligned}
$$

To prove the proposition we must show that (8) holds for all $w \in C_{0}^{\infty}\left(\boldsymbol{R}_{+}, F_{0}\right)$ if and only if (12) holds for all $u$ with compact support in $U \cap M$.

First assume that (8) holds, let $\xi^{\prime} \neq 0$, and apply (8) to $w(t)=\tilde{u}\left(\xi^{\prime}, t /\left|\xi^{\prime}\right|\right)$ with $\zeta^{\prime}=\xi^{\prime} /\left|\xi^{\prime}\right|$. Then with a new variable of integration $x^{n}=t /\left|\xi^{\prime}\right|$ we obtain

$$
\begin{aligned}
&\left|\xi^{\prime}\right|^{2} \int_{0}^{\infty}\left|\tilde{u}\left(\xi^{\prime}, x^{n}\right)\right|^{2} d x^{n}+\int_{0}^{\infty}\left|D_{n} \tilde{u}\left(\xi^{\prime}, x^{n}\right)\right|^{2} d x^{n} \\
& \leq c\left\{\int_{0}^{\infty}\left|a\left(0, \xi^{\prime}, D_{n}\right) * \tilde{u}\left(\xi^{\prime}, x^{n}\right)\right|^{2} d x^{n}+\int_{0}^{\infty}\left|b\left(0, \xi^{\prime}, D_{n}\right) \tilde{u}\left(\xi^{\prime}, x^{n}\right)\right|^{2} d x^{n}\right. \\
&\left.+\left|\xi^{\prime}\right|\left|a(0,0,1)^{*} \tilde{u}\left(\xi^{\prime}, 0\right)\right|^{2}\right\},
\end{aligned}
$$

and integration with respect to $\xi^{\prime}$ produces (12).

Now assume that (12) holds for all $u$ with support in $M \cap U$, let $w \in C_{0}^{\infty}\left(\boldsymbol{R}_{+}, F_{0}\right)$, and $\left|\boldsymbol{\zeta}^{\prime}\right|=1$. Choose $\varphi \in C_{0}^{\infty}\left(\boldsymbol{R}^{n-1}\right)$ with $\int\left|\varphi\left(x^{\prime}\right)\right|^{2} d x^{\prime}=(2 \pi)^{-n-1}$ and define

$$
u_{\varepsilon}(x)=\varepsilon^{1 / 2} w\left(x^{n} / \varepsilon\right) \varphi\left(x^{\prime}\right) e^{i\left\langle\zeta^{\prime}, x^{\prime}\right\rangle / \varepsilon}
$$

for $x=\left(x^{\prime}, x^{n}\right) \in \boldsymbol{R}^{n-1} \times \boldsymbol{R}_{+}$and $\varepsilon>0$. If $\varepsilon$ is sufficiently small and the support 
of $\varphi$ is small enough, then $u_{\varepsilon}$ has support in $U$ and hence (12) holds with $\tilde{u}$ replaced by $\tilde{u}_{\varepsilon}$. The estimate $(8)$ now follows by letting $\varepsilon \rightarrow 0$. In fact,

$$
\begin{aligned}
\tilde{u}_{\varepsilon}\left(\xi^{\prime}, x^{n}\right) & =\varepsilon^{1 / 2} w\left(x^{n} / \varepsilon\right) \tilde{\varphi}\left(\xi^{\prime}-\zeta^{\prime} / \varepsilon\right), \\
b\left(0, \xi^{\prime}, D_{n}\right) \tilde{u}_{\varepsilon}\left(\xi^{\prime}, x^{n}\right) & =\varepsilon^{-1 / 2} b\left(0, \varepsilon \xi^{\prime}, D_{n}\right) w\left(x^{n} / \varepsilon\right) \tilde{\varphi}\left(\xi^{\prime}-\zeta^{\prime} / \varepsilon\right),
\end{aligned}
$$

and hence

$$
\begin{aligned}
\iint_{0}^{\infty}\left|b\left(0, \xi^{\prime}, D_{n}\right) \tilde{u}_{\varepsilon}\left(\xi^{\prime}, x^{n}\right)\right|^{2} d \xi^{\prime} d x^{n} \\
=\varepsilon^{-n-1} \iint_{0}^{\infty}\left|b\left(0, \xi^{\prime}, D_{n}\right) w(t)\right|^{2}\left|\tilde{\varphi}\left(\frac{\xi^{\prime}-\zeta^{\prime}}{\varepsilon}\right)\right|^{2} d \xi^{\prime} d t
\end{aligned}
$$

which converges to

$$
\int_{0}^{\infty}\left|b\left(0, \zeta^{\prime}, D_{n}\right) w(t)\right|^{2} d t
$$

as $\varepsilon \rightarrow 0$. The other terms in (12) are treated similarly, and the proof is complete.

Proposition 4. There exists a constant $C$ such that (8) holds for all unit vectors $\zeta^{\prime} \in \boldsymbol{R}^{n-1}$ and all $w \in C_{0}^{\infty}\left(\boldsymbol{R}_{+}, F_{0}\right)$ if and only if for each $\xi^{\prime} \in \boldsymbol{R}^{n-1}-\{0\}$ the system of equations

$$
\begin{array}{ll}
a\left(0, \xi^{\prime}, D_{t}\right) *(t)=0, & t \geq 0, \\
b\left(0, \xi^{\prime}, D_{t}\right) w(t)=0, & t \geq 0, \\
a(0,0,1) * w(0)=0 &
\end{array}
$$

has no nonzero solution $w$ in $L_{2}\left(\boldsymbol{R}_{+}, F_{0}\right)$.

Proof. For $\xi^{\prime} \in \boldsymbol{R}^{n-1}$ and $\zeta_{n} \in \boldsymbol{C}$ define

$$
\begin{aligned}
\Delta\left(\xi^{\prime}, \zeta_{n}\right) & =a\left(0, \xi^{\prime}, \zeta_{n}\right) a\left(0, \xi^{\prime}, \bar{\zeta}_{n}\right)^{*}+b\left(0, \xi^{\prime}, \bar{\zeta}_{n}\right)^{*} b\left(0, \xi^{\prime}, \zeta_{n}\right) \\
\Delta\left(\xi^{\prime}, D_{t}\right) & =a\left(0, \xi^{\prime}, D_{t}\right) a\left(0, \xi^{\prime}, D_{t}\right)^{*}+b\left(0, \xi^{\prime}, D_{t}\right)^{*} b\left(0, \xi^{\prime}, D_{t}\right) .
\end{aligned}
$$

Since (2) is exact for all real $\zeta=\left(\xi^{\prime}, \zeta_{n}\right) \neq 0$, it follows that $\Delta\left(\xi^{\prime}, \zeta_{n}\right)$ is nonsingular when $\zeta_{n}$ is real; and since $\Delta\left(\xi^{\prime}, \zeta_{n}\right)^{*}=\Delta\left(\xi^{\prime}, \bar{\zeta}_{n}\right)$, it follows that the solutions $\zeta_{n} \in C$ to the equation $\operatorname{det} \Delta\left(\xi^{\prime}, \zeta_{n}\right)=0$ occur in conjugate pairs. This means that each solution to the differential equation

$$
\Delta\left(\xi^{\prime}, D_{t}\right) w(t)=0, \quad t \geq 0,
$$


can be written as a sum of two solutions $w^{\prime}$ and $w^{\prime \prime}$, where $w^{\prime}(t)$ decreases exponentially and $w^{\prime \prime}(t)$ increases exponentially as $t$ tends to $\infty$; and, moreover, the set of all exponentially decreasing solutions to (14) forms a vector space of the same dimension as $F_{0}$.

Now assume that (13) has no nontrivial solution in $L_{2}\left(\boldsymbol{R}_{+}, F_{0}\right)$, and note that the same is true for the system

$$
\begin{aligned}
& \Delta\left(\xi^{\prime}, D_{t}\right) w(t)=0, \quad t \geq 0, \\
& \left(a(0,0,1) a(0,0,1)^{*}+b(0,0,1) * b\left(0, \xi^{\prime}, D_{t}\right)\right) w(0)=0 .
\end{aligned}
$$

In fact, if $w$ is an $L_{2}$ solution to (15), then $w$ must be $C^{\infty}$ and exponentially decreasing as $t$ tends to $\infty$. Since $b(0,0,1) a(0,0,1)=0$, the mappings $a(0,0,1)$ and $b(0,0,1)^{*}$ map into orthogonal subspaces of $F_{0}$, and hence the second line in (15) is equivalent to

$$
a(0,0,1)^{*} w(0)=0, \quad b(0,0,1)^{*} b\left(0, \xi^{\prime}, D_{t}\right) w(0)=0 .
$$

These boundary conditions allow us to integrate by parts to obtain

$$
\begin{aligned}
0 & =\int_{0}^{\infty}\left\langle\Delta\left(\xi^{\prime}, D_{t}\right) w(t), w(t)\right\rangle d t \\
& =\int_{0}^{\infty}\left|a\left(0, \xi^{\prime}, D_{t}\right)^{*} w(t)\right|^{2} d t+\int_{0}^{\infty}\left|b\left(0, \xi^{\prime}, D_{t}\right) w(t)\right|^{2} d t
\end{aligned}
$$

so that $a\left(0, \xi^{\prime}, D_{t}\right) w$ and $b\left(0, \xi^{\prime}, D_{t}\right) w$ vanish, and hence $w$ is an $L_{2}$ solution to (13). Thus, by our assumption, $w$ must be identically 0 .

Since (15) has no nontrivial solution in $L_{2}\left(\boldsymbol{R}_{+}, F_{0}\right)$, this system satisfies the hypotheses of Theorem 10.2.1 in [6], and thus for any $\boldsymbol{w} \in C_{0}^{\infty}\left(\boldsymbol{R}_{+}, F_{0}\right)$ there exists $v \in C^{\infty}\left(\boldsymbol{R}_{+}, F_{0}\right)$ such that

$$
\begin{aligned}
& \Delta\left(\zeta^{\prime}, D_{t}\right) v(t)=w(t), \quad t \geq 0 \\
& a(0,0,1)^{*} v(0)=0, \\
& b(0,0,1)^{*} b\left(0, \zeta^{\prime}, D_{t}\right) v(0)=0
\end{aligned}
$$

and

$$
\int_{0}^{\infty}\left(|v(t)|^{2}+\left|D_{t} v(t)\right|^{2}+\left|D_{t}^{2} v(t)\right|^{2}\right) d t \leq C \int_{0}^{\infty}|w(t)|^{2} d t
$$

where the constant $C$ does not depend on $w$ or on $\zeta^{\prime} \in R^{n-1}$ with $\left|\zeta^{\prime}\right|=1$. If $w$ satisfies $a(0,0,1) * w(0)=0$, then we may integrate by parts to obtain 


$$
\begin{aligned}
\int_{0}^{\infty}|w(t)|^{2} d t= & \int_{0}^{\infty}\left\langle w(t), \Delta\left(\zeta^{\prime}, D_{t}\right) v(t)\right\rangle d t \\
& +\int_{0}^{\infty}\left\langle a\left(0, \zeta^{\prime}, D_{t}\right) * w(t), a\left(0, \zeta^{\prime}, D_{t}\right) * v(t)\right\rangle d t \\
& +\int_{0}^{\infty}\left\langle b\left(0, \zeta^{\prime}, D_{t}\right) w(t), b\left(0, \zeta^{\prime}, D_{t}\right) v(t)\right\rangle d t
\end{aligned}
$$

and using (16) and Schwarz's inequality we get

$$
\begin{aligned}
\int_{0}^{\infty}|w(t)|^{2} d t \leq C & \left\{\int_{0}^{\infty}\left|a\left(0, \zeta^{\prime}, D_{t}\right) * w(t)\right|^{2} d t\right. \\
& \left.+\int_{0}^{\infty}\left|b\left(0, \zeta^{\prime}, D_{t}\right) w(t)\right|^{2} d t\right\}^{1 / 2}\left\{\int_{0}^{\infty}|w(t)|^{2} d t\right\}^{1 / 2} .
\end{aligned}
$$

The inequality

$$
\int_{0}^{\infty}|w(t)|^{2} d t \leq C\left\{\int_{0}^{\infty}\left|a\left(0, \zeta^{\prime}, D_{t}\right) * w(t)\right|^{2} d t+\int_{0}^{\infty}\left|b\left(0, \zeta^{\prime}, D_{t}\right) w(t)\right|^{2} d t\right\}
$$

follows immediately, and since

$$
\begin{aligned}
\left|D_{t} w(t)\right|^{2} & \leq C\left\{\left|a(0,0,1) * D_{t} w(t)\right|^{2}+\left|b(0,0,1) D_{t} w(t)\right|^{2}\right\} \\
& \leq C\left\{\left|a\left(0, \zeta^{\prime}, D_{t}\right) * w(t)\right|^{2}+\left|b\left(0, \zeta^{\prime}, D_{t}\right) w(t)\right|^{2}+|w(t)|^{2}\right\},
\end{aligned}
$$

we now have a constant $C$ such that (8) holds for all unit vectors $\zeta^{\prime} \in \boldsymbol{R}^{n-1}$ and all $w \in C_{0}^{\infty}\left(\boldsymbol{R}_{+}, F_{0}\right)$ satisfying $a(0,0,1)^{*} w(0)=0$. It is now a fairly easy matter to obtain (8) for all unit vectors $\zeta^{\prime} \in \boldsymbol{R}^{n-1}$ and all $w \in C_{0}^{\infty}\left(\boldsymbol{R}_{+}, F_{0}\right)$; one need only repeat the argument made in the proof of Proposition 1.

To complete the proof of Proposition 4 assume that the estimate (8) holds for all $\left|\zeta^{\prime}\right|=1$ and all $w \in C_{0}^{\infty}\left(\boldsymbol{R}_{+}, F_{0}\right)$, and let $u$ be an $L_{2}$ solution to (13). Then $u$ is $C^{\infty}$ exponentially decreasing. If we choose $\varphi \in C_{0}^{\infty}(\boldsymbol{R})$ which is 1 on a neighborhood of 0 , then (8) must hold for $\zeta^{\prime}=\zeta^{\prime} /\left|\zeta^{\prime}\right|$ and for $w(t)=$ $\varphi(t / n) u\left(t /\left|\zeta^{\prime}\right|\right)$. Letting $n \rightarrow \infty$, we obtain

$$
\int_{0}^{\infty}|u(t)|^{2} d t \leq 0
$$

so that $u$ is identically 0 , as required.

For a vector space $V$ we shall denote the intersection $C^{\infty}\left(\boldsymbol{R}_{+}, V\right) \cap L_{2}\left(\boldsymbol{R}_{+}, V\right)$ by $C^{\infty} L_{2}\left(\boldsymbol{R}_{+}, V\right)$, and in the context

$$
C^{\infty} L_{2}\left(\boldsymbol{R}_{+}, E_{0}\right) \stackrel{a\left(0, \zeta^{\prime}, D_{t}\right)}{\longrightarrow} C^{\infty} L_{2}\left(\boldsymbol{R}_{+}, F_{0}\right) \stackrel{b\left(0, \zeta^{\prime}, D_{t}\right)}{\longrightarrow} C^{\infty}\left(\boldsymbol{R}_{+}, G_{0}\right)
$$


we shall understand $a\left(0, \zeta^{\prime}, D_{t}\right)$ to be the unbounded operator whose domain consists of all $w \in C^{\infty} L_{2}\left(\boldsymbol{R}_{+}, E_{0}\right)$ such that $a\left(0, \zeta^{\prime}, D_{t}\right) w \in C^{\infty} L_{2}\left(\boldsymbol{R}_{+}, F_{0}\right)$.

Proposition 5. The system (13) has no nonzero solution in $L_{2}$ if and only if the sequence (17) is exact.

Proof. If $w$ is a nonzero $L_{2}$ solution to (13), then $b\left(0, \zeta^{\prime}, D_{t}\right) w=0$, and the other two equations in (13) can be used to obtain

$$
\int_{0}^{\infty}\left\langle w(t), a\left(0, \zeta^{\prime}, D_{t}\right) u(t)\right\rangle d t=\int_{0}^{\infty}\left\langle a\left(0, \zeta^{\prime}, D_{t}\right)^{*} w(t), u(t)\right\rangle d t=0,
$$

so that $w$ is orthogonal to the range of $a\left(0, \zeta^{\prime}, D_{t}\right)$ and hence defines a nonzero homology class for (17).

On the other hand, if (13) has no nonzero solution in $L_{2}$, then, as we have seen, the same is true for the system (15), and by Theorem 10.2.1 in [6] we can write any $w \in C^{\infty} L_{2}\left(R_{+}, F_{0}\right)$ as

$$
w=a\left(0, \zeta^{\prime}, D_{t}\right) a\left(0, \zeta^{\prime}, D_{t}\right) * v+b\left(0, \zeta^{\prime}, D_{t}\right) * b\left(0, \zeta^{\prime}, D_{t}\right) v
$$

where $v \in C^{\infty}\left(\boldsymbol{R}_{+}, F_{0}\right)$ satisfies $\int_{0}^{\infty}\left(\left|D_{t} v(t)\right|^{2}+|v(t)|^{2}\right) d t<\infty$ and $b(0,0,1)^{*}$. $b\left(0, \zeta^{\prime}, D_{t}\right) v(0)=0$. This last condition allows us to integrate by parts and to obtain

$$
\begin{aligned}
\int_{0}^{\infty}\left\langle b\left(0, \zeta^{\prime}, D_{t}\right) * b\left(0, \zeta^{\prime}, D_{t}\right) v(t), u(t)\right\rangle d t \\
\quad=\int_{0}^{\infty}\left\langle b\left(0, \zeta^{\prime}, D_{t}\right) v(t), b\left(0, \zeta^{\prime}, D_{t}\right) u(t)\right\rangle d t
\end{aligned}
$$

and it follows that the second term on the right of (18) is always orthogonal to the kernel of $b\left(0, \zeta^{\prime}, D_{t}\right)$. Now the first term on the right of (18) is always in the kernel of $b\left(0, \zeta^{\prime}, D_{t}\right)$, and hence if $w$ is also in this kernel, it follows that

$$
w=a\left(0, \zeta^{\prime}, D_{t}\right) a\left(0, \zeta^{\prime}, D_{t}\right) * v .
$$

In other words, (17) is exact.

From Propositions 3, 4, and 5 it follows that the estimate

$$
\|u\|_{1} \leq c\left\{\left\|A^{*} u\right\|+\|B u\|+\|u\|+{ }^{\partial}\left\|a(d r)^{*} u\right\|_{1 / 2}\right\}
$$

holds for all $u$ in a neighborhood of $0 \in \partial M$ if and only if the sequence (17) is exact for all $\zeta^{\prime} \in \boldsymbol{R}^{n-1}-\{0\}$. Although it was obtained using a specially chosen coordinate, the condition that (17) be exact for all $\zeta^{\prime} \in \boldsymbol{R}^{n-1}-\{0\}$ makes sense independently of any such choice. In fact, in (17) we may let 0 be any point 
$x$ on $\partial M$, and $\left(0, \zeta^{\prime}, 0\right)$ may be considered as an arbitrary element $\zeta^{\prime} \in T_{x}^{*}(M)$ which is orthogonal to $d r$; the operator $a\left(0, \zeta^{\prime}, D_{t}\right)$ may then be denoted by

$$
a\left(\zeta^{\prime}+d r D_{t}\right)=a\left(\zeta^{\prime}\right)+a(d r) D_{t}
$$

and this is a well-defined operator from $C^{\infty}\left(\boldsymbol{R}_{+}, E_{x}\right)$ to $C^{\infty}\left(\boldsymbol{R}_{+}, F_{x}\right)$, independent of any choice of coordinate or of basis in $E_{x}$ and $F_{x}$. We have now that the estimate (5) holds for all $u$ in a neighborhood of $x \in \partial M$ if and only if the sequence

$$
C^{\infty} L_{2}\left(\boldsymbol{R}_{+}, E_{x}\right) \stackrel{a\left(\zeta^{\prime}+d r D_{t}\right)}{\longrightarrow} C^{\infty} L_{2}\left(\boldsymbol{R}_{+}, F_{x}\right) \stackrel{b\left(\zeta^{\prime}+d r D_{t}\right)}{\longrightarrow} C^{\infty} L_{2}\left(\boldsymbol{R}_{+}, G_{x}\right)
$$

is exact for all $\zeta^{\prime} \in T_{x}^{*}(M)$ which are orthogonal to $d r$. Now if $\xi$ is any element of $T_{x}^{*}(M)$ such that $\xi \wedge d r \neq 0$, then we can write $\xi=\xi^{\prime}+\lambda d r$ where $\xi^{\prime}$ is orthogonal to $d r$ and $\lambda$ is a real number. Since

$$
a\left(\xi+d r D_{t}\right)=a\left(\xi^{\prime}+d r\left(D_{t}+\lambda\right)\right)=e^{-i \lambda t} a\left(\xi^{\prime}+d r D_{t}\right) e^{i \lambda t}
$$

we find that (19) is exact for all $\zeta^{\prime}$ orthogonal to $d r$ if and only if it is exact for all $\zeta^{\prime}$ with $\zeta^{\prime} \wedge d r \neq 0$. We have now proved the following theorem.

Theorem 1. The coercive estimate

$$
\|u\|_{1} \leq c_{M}\left\{\left\|A^{*} u\right\|+\|B u\|+\|u\|\right\}
$$

holds for all $u \in C^{\infty}(M, F)$ satisfying

$$
a(d r)^{*} u=0 \quad \text { on } \partial M,
$$

if and only if the sequence (19) is exact for each $x \in \partial M$ and each cotangent vector $\zeta^{\prime} \in T_{x}^{*}(M)$ satisfying $\zeta^{\prime} \wedge d r \neq 0$.

Theorem 2. The coercive estimate (3) holds for all $u \in C^{\infty}(M, F)$ satisfying (4) and for all $M \subset M^{\prime}$ if and only if the sequence

$$
C^{\infty} L_{2}\left(\boldsymbol{R}, E_{x}\right) \stackrel{a\left(\xi+\eta D_{t}\right)}{\longrightarrow} C^{\infty} L_{2}\left(\boldsymbol{R}_{+}, F_{x}\right) \stackrel{b\left(\xi+\eta D_{t}\right)}{\longrightarrow} C^{\infty} L_{2}\left(\boldsymbol{R}_{+}, G_{x}\right)
$$

is exact for each $x \in M^{\prime}$ and each pair of real cotangent vectors $\xi, \eta \in T_{x}^{*}\left(M^{\prime}\right)$ with $\xi \wedge \eta \neq 0$.

Proof. Theorem 2 follows immediately from Theorem 1 when we note that if $\eta \in T_{x}^{*}\left(M^{\prime}\right)$ and $\eta \neq 0$, then there exists $M \subset M^{\prime}$ such that $x \in \partial M$ and such that $\eta$ is normal to $M$.

Note that the conditions for the coercive estimate given in these theorems do not depend on the Riemannian metric on $M^{\prime}$ nor on the Hermitian structures on $E, F$, and $G$. 


\section{Local exactness}

In this section we assume that

$$
E \stackrel{A}{\longrightarrow} F \stackrel{B}{\longrightarrow} G
$$

is a segment of the Spencer sequence corresponding to a formally integrable differential operator on $M^{\prime}$. (For definitions see Spencer [9], particularly Definition 1.2.7 and Theorem 1.6.1.) We continue to assume that the symbol sequence of (1) is exact for real cotangent vectors. We shall prove:

Theorem 3. Assume that for each $M \subset M^{\prime}$ the coercive estimate (3) holds for all $u \in C^{\infty}(M, F)$ satisfying (4). Then the sequence (1) is locally exact; in fact, if $x$ is any point in $M^{\prime}$, then every neighborhood of $x$ contains a manifoldwith-boundary $M$ such that any $v \in C^{\infty}(M, F)$ satisfying $B v=0$ can be expressed as $v=A u$ for some $u \in C^{\infty}(M, E)$.

In view of Theorem 2 both the hypotheses and the conclusion of Theorem 3 are local in character and do not depend on any choice of Riemannian metric or Hermitian inner product. Hence we may assume that $M^{\prime}$ is an open subset of $\boldsymbol{R}^{n}$, that $E, F$, and $G$ are product bundles, and that the differential operators are given by

$$
\begin{aligned}
A & =\sum A_{j}(x) D_{j}+A_{0}(x) \\
A^{*} & =\sum A_{j}(x)^{*} D_{j}+A_{0}(x)^{*}, \\
B & =\sum B_{j}(x) D_{j}+B_{0}(x)
\end{aligned}
$$

where the coefficients $A_{j}(x)$ and $B_{j}(x)$ are $C^{\infty}$ matrix-valued functions of $x$. To prove the theorem it will suffice to show that (1) is exact for sections over $M_{s}=\left\{x \in \boldsymbol{R}^{n}|| x \mid \leq s\right\}$ if $s>0$ is sufficiently small.

The proof will proceed in two steps. We first treat the case where $A$ and $B$ have constant coefficients, using an argument communicated to the author by J.J.Kohn, and next we use a shrinking argument to reduce the general case to the case of constant coefficients.

Assume that $A$ and $B$ have constant coefficients. Since the coercive estimate (3) holds for sections over $M_{s}$, the Neumann problem can be solved there, and it suffices to prove that every harmonic $v \in C^{\infty}\left(M_{s}, F\right)$ can be written as $v=A u$. (For details here see Spencer [9, pp. 231-233] or Sweeney [10, pp. 251-254].) But, by definition, each harmonic $v$ solves the elliptic boundary value problem

$$
\begin{aligned}
\left(A A^{*}+B^{*} B\right) v & =0 & & \text { on } M_{s}, \\
\left(a(d r) a(d r)^{*}+b(d r)^{*} B\right) v & =0 & & \text { on } \partial M_{s},
\end{aligned}
$$

which in the present case has analytic coefficients. Hence by a theorem of C. B. Morrey, Jr. and L. Nirenberg (see [8] or Y. Kato [7, Theorem 4]) $v$ extends 
to an analytic function on $M_{s^{\prime}}$ for some $s^{\prime}>s$, and by the uniqueness of analytic continuation $B v=0$ on $M_{s^{\prime}}$. Since (1) is a segment of a Spencer sequence, it is formally exact, and according to a theorem of L. Ehrenpreis and B. Malgrange (see Hörmander [5, Theorem 7.6.13]) there exists a $C^{\infty} u$ on the interior of $M_{s^{\prime}}$ such that $A u=v$. The restriction of $u$ to $M_{s}$ is thus the desired element of $C^{\infty}\left(M_{s}, E\right)$, and in the constant coefficient case the proof of Theorem 3 is complete.

Returning to the general case, we define differential operators

$$
\begin{aligned}
A^{s} & =\sum A_{j}(s x) D_{j}+s A_{0}(s x), \\
B^{s} & =\sum B_{j}(s x) D_{j}+s B_{0}(s x),
\end{aligned}
$$

for $0 \leq s \leq 1$, and we note that the change of coordinate $x=y / s$ carries $A^{s}$ and $B^{s}$ over into $s A$ and $s B$ respectively. Thus in order to prove that (1) is exact for sections over $M_{s}$ for small $s$ it suffices to prove that the sequence

$$
C^{\infty}\left(M_{1}, E\right) \stackrel{A^{s}}{\longrightarrow} C^{\infty}\left(M_{1}, F\right) \stackrel{B^{s}}{\longrightarrow} C^{\infty}\left(M_{1}, G\right)
$$

is exact for small $s>0$.

The operators $A^{0}$ and $B^{0}$ have constant coefficients, and Theorem 2 shows that the coercive estimate

$$
\|u\|_{1} \leq c\left\{\left\|A^{0 *} u\right\|+\left\|B^{0} u\right\|+\|u\|+{ }^{\circ}\left\|a^{0}(d r)^{*} u\right\|_{1 / 2}\right\}
$$

holds. Since $A^{0}$ and $B^{0}$ have no zero order term, it is easy to check that

$$
E \stackrel{A^{0}}{\longrightarrow} F \stackrel{B^{0}}{\longrightarrow} G
$$

continues to be part of the Spencer sequence associated with a formally integrable differential operator. (See Spencer [9, Theorem 1.6.1].) By the argument given above it follows that the sequence (25) is exact for sections over $M_{1}$ and also that the harmonic space for the Neumann problem over $M_{1}$ vanishes. An application of the closed graph theorem now yields

$$
\|u\|_{1} \leq c\left\{\left\|A^{0 *} u\right\|+\left\|B^{0} u\right\|+{ }^{\partial}\left\|a^{0}(d r)^{*} u\right\|_{1 / 2}\right\}
$$

for all $u \in C^{\infty}\left(M_{1}, F\right)$. But given $\varepsilon>0$ we can, in view of (22), choose $s>0$ so small that

$$
\begin{gathered}
\left\|A^{0 *} u-A^{s *} u\right\| \leq \varepsilon\|u\|_{1}, \quad\left\|B^{0} u-B^{s} u\right\| \leq \varepsilon\|u\|_{1}, \\
{ }^{\partial}\left\|a^{0}(d r)^{*} u-a^{s}(d r)^{*} u\right\|_{1 / 2} \leq \varepsilon^{\partial}\|u\|_{1 / 2} \leq \varepsilon c\|u\|_{1}
\end{gathered}
$$

these inequalities with $\varepsilon>0$ sufficiently small combine with (26) to yield

$$
\|u\|_{1} \leq c\left\{\left\|A^{s *} u\right\|+\left\|B^{s} u\right\|+{ }^{\partial}\left\|a^{s}(d r)^{*} u\right\|_{1 / 2}\right\}
$$


for $s>0$ sufficiently small. It now follows that the harmonic space for the Neumann problem for (23) vanishes, and hence (23) is exact for $s>0$ sufficiently small. The proof of Theorem 3 is complete.

\section{A sufficient condition for coerciveness}

We now give an explicitly algebraic condition which implies the coercive estimate.

Theorem 4. For each $x \in \partial M$ assume that the symbol sequence (2) is exact for all nonzero $\zeta \in T_{x}^{*}(M) \otimes C$ of the form $\zeta=\xi^{\prime}+\lambda d r$, where $\xi^{\prime} \in T_{x}^{*}(M)$ and $\lambda \in C$. Then the coercive estimate (3) holds for all $u \in C^{\infty}(M, F)$ satisfying (4).

Proof. Let $x_{0} \in \partial M$ and choose a local coordinate and special frames near $x_{0}$ as in $\S 1$. Then by Proposition 4 the theorem will be proved if we can show that the system

$$
\begin{array}{rlrl}
a\left(0, \xi^{\prime}, D_{t}\right) * w(t) & =0, & & t \geq 0, \\
b\left(0, \xi^{\prime}, D_{t}\right) w(t) & =0, & & t \geq 0, \\
a(0,0,1) * w(0) & =0, &
\end{array}
$$

has no nonzero solution in $L_{2}\left(\boldsymbol{R}_{+}, F_{0}\right)$.

So let $w$ be a nonzero $L_{2}$ solution to (13), and recall that $w$ must then satisfy the differential equation (14). It follows that

$$
w(t)=\sum p_{j}(t) \exp \left(i \lambda_{j} t\right), \quad t \geq 0,
$$

where the $\lambda_{j}$ 's are the solutions to $\operatorname{det} \Delta\left(\xi^{\prime}, \lambda\right)=0$ having positive imaginary part. Now

$$
0=b\left(0, \xi^{\prime}, D_{t}\right) w(t)=\sum\left[b\left(0, \xi^{\prime}, \lambda_{j}\right) p_{j}(t)+b\left(0,0, D_{t}\right) p_{j}(t)\right] \exp \left(i \lambda_{j} t\right)
$$

and we claim that

$$
\begin{aligned}
b\left(0, \xi^{\prime}, D_{t}\right)\left(p_{j}(t) \exp \left(i \lambda_{j} t\right)\right) \\
\quad=\left(b\left(0, \xi^{\prime}, \lambda_{j}\right) p_{j}(t)+b\left(0,0, D_{t}\right) p_{j}(t)\right) \exp \left(i \lambda_{j} t\right)=0
\end{aligned}
$$

for each $j$. In fact, we may assume that the $\lambda_{j}$ s are all different and that $\left|\lambda_{j}\right| \geq\left|\lambda_{j+1}\right|$ for each $j$. Then there exists a complex number $\omega$ such that $\operatorname{Re}\left\{\lambda_{1} \omega\right\}>\operatorname{Re}\left\{\lambda_{j} \omega\right\}$ for each $j>1$. Now since the right side of (28) is an analytic function of $t,(28)$ must hold for all $t \in C$ and, in particular, for $t=s \omega$, where $s$ is real. To obtain (29) for $j=1$ substitute $s \omega$ for $t$, multiply by $\exp \left(-i \lambda_{1} \omega s\right)$, and let $s \rightarrow+\infty$. Several repetitions of this argument yield (29) for all $j$.

Using (29) we shall now show that there exist $E_{0}$-valued polynomials $q_{j}(t)$ such that 


$$
a\left(0, \xi^{\prime}, D_{t}\right)\left(q_{j}(t) \exp \left(i \lambda_{j} t\right)\right)=p_{j}(t) \exp \left(i \lambda_{j} t\right) .
$$

In fact, if $p_{j k} t^{k}$ is the highest order term occurring in $p_{j}(t)$, then by looking at the coefficient of $t^{k}$ in (29) we obtain

$$
b\left(0, \xi^{\prime}, \lambda_{j}\right) p_{j k}=0 .
$$

Since the symbol sequence is exact for $\zeta=\left(\xi^{\prime}, \lambda_{j}\right)$, there exists $q_{j k} \in E_{0}$ such that $a\left(0, \xi^{\prime}, \lambda_{j}\right) q_{j k}=p_{j k}$, and it follows that

$$
a\left(0, \xi^{\prime}, D_{t}\right)\left(q_{j k} t^{t} \exp \left(i \lambda_{j} t\right)\right)=p_{j}(t) \exp \left(i \lambda_{j} t\right)+r(t) \exp \left(i \lambda_{j} t\right),
$$

where $r(t)$ is a polynomial of order $<k$. If $k=0$, we have thus found the required $q_{j}(t)$; and if $k>0$, we have reduced the problem to the case where $p_{j}(t)$ has order $\leq k-1$. The existence of $p_{j}(t)$ is thus established by induction on $k$.

Now let $u(t)=\sum q_{j}(t) \exp \left(i \lambda_{j} t\right)$, and notice that $u \in L_{2}\left(\boldsymbol{R}_{+}, E_{0}\right)$ and $a\left(0, \xi^{\prime}, D_{t}\right) u(t)=w(t)$ for $t \geq 0$. Using the boundary condition $a(0,0,1) * w(0)$ $=0$, we may integrate by parts to obtain

$$
\int_{0}^{\infty}|w(t)|^{2} d t=\int_{0}^{\infty}\left\langle w(t), a\left(0, \xi^{\prime}, D_{t}\right) u(t)\right\rangle d t+\int_{0}^{\infty}\left\langle a\left(0, \xi^{\prime}, D_{t}\right)^{*} w(t), u(t)\right\rangle d t .
$$

In view of the first equation in (13) the last integral vanishes, and hence $w(t)=0$ for all $t \geq 0$.

\section{The Cohen-Macaulay case}

Let

$$
0 \longrightarrow E^{0} \stackrel{D}{\longrightarrow} E^{1} \stackrel{D}{\longrightarrow} \cdots \stackrel{D}{\longrightarrow} E^{N} \longrightarrow 0
$$

be the Spencer sequence associated with an elliptic, formally integrable differential operator on $M^{\prime}$, and let

$$
0 \longrightarrow E_{x}^{0} \stackrel{a(\zeta)}{\longrightarrow} E_{x}^{1} \stackrel{a(\zeta)}{\longrightarrow} \cdots \stackrel{a(\zeta)}{\longrightarrow} E_{x}^{N} \longrightarrow 0
$$

be its symbol sequence. Let $\mathscr{V}_{x}$ denote the complex characteristic variety of (31) at $x$. Thus $\mathscr{V}_{x}$ consists of all $\zeta \in T_{x}^{*}\left(M^{\prime}\right) \otimes C$ for which $a(\zeta): E_{x}^{0} \rightarrow E_{x}^{1}$ has a nonzero kernel, and using the properties of Spencer sequences, one can show that $q=\operatorname{codim} \mathscr{V}_{x}$ is a constant function of $x$.

In this section we shall assume that (32) is exact at $E_{x}^{q+1}, \cdots, E_{x}^{N}$ for every nonzero $\zeta \in T_{x}^{*}\left(M^{\prime}\right) \otimes C$ and every $x \in M^{\prime}$. A theorem due to D. G. Quillen (see Spencer [9, Theorem 1.7.2]) asserts that (32) is exact at every position if $\zeta \notin \mathscr{V}_{x}$ so this amounts to an assumption only on the points in $\mathscr{V}=\cup \mathscr{V}_{x}$. As noted by V. W. Guillemin and S. Sternberg [4], this assumption is equivalent 
to requiring that each $\zeta \in \mathscr{V}_{x}$ be a Cohen-Macaulay point for the symbol module of (31) at $x$.

Theorem 5. Assume that every $\zeta \in \mathscr{V}$ is a Cohen-Macaulay point as described above. Then (31) is locally exact at the positions $E^{q}, \cdots, E^{N}$, and the coercive estimate holds at these positions for any $M \subset M^{\prime}$. If $0 \leq s<q$, then the coercive estimate holds at $E^{s}$ if and only if the projection ${ }^{\partial} \mathscr{V}_{x}$ of $\mathscr{V}_{x}$ into $T^{*}(\partial M) \otimes C$ satisfies ${ }^{\partial} \mathscr{V}_{x} \cap T_{x}^{*}(\partial M)=0$ for each $x \in \partial M$.

The main ingredient of our proof here is a canonical form theorem due to W. V. Guillemin and S.Sternberg. (See Theorem 2 in [4].) Namely, under the assumptions made here Guillemin and Sternberg have proved that every $\zeta \in \mathscr{V}$ has a conical neighborhood $U \subset T^{*}\left(M^{\prime}\right) \otimes C$ over which there exist invertible formal pseudo-differential operators $R: E^{s} \rightarrow E^{s}$ (for definitions see $\S 2$ in [3]) such that the complex

$$
0 \longrightarrow E^{0} \stackrel{R D R^{-1}}{\longrightarrow} E^{1} \stackrel{R D R^{-1}}{\longrightarrow} E^{2} \stackrel{R D R^{-1}}{\longrightarrow} \cdots
$$

is the direct sum of two pseudo-differential complexes

$$
\begin{gathered}
0 \longrightarrow E^{\prime 0} \stackrel{D^{\prime}}{\longrightarrow} E^{\prime 1} \stackrel{D^{\prime}}{\longrightarrow} E^{\prime 2} \longrightarrow \cdots, \\
0 \longrightarrow E^{\prime \prime 0} \stackrel{D^{\prime \prime}}{\longrightarrow} E^{\prime \prime 1} \stackrel{D^{\prime \prime}}{\longrightarrow} E^{\prime \prime 2} \stackrel{D^{\prime \prime}}{\longrightarrow} \cdots,
\end{gathered}
$$

where the symbol sequence of (34) is exact at each $\zeta \in U$ and (35) is a Poincaré complex. To say that (35) is a Poincaré complex means that there exist a product bundle $F$ over $U$, a vector space $W$ with basis $\left\{\boldsymbol{w}^{1}, \ldots, \boldsymbol{w}^{q}\right\}$, and commuting formal pseudo-differential operotors $A_{1}: F \rightarrow F, \cdots, A_{q}: F \rightarrow F$ such that

$$
E^{\prime s}=\Lambda^{s} W \otimes F, \quad D^{\prime \prime} u=\sum w^{i} \wedge A_{i} u .
$$

In [4] Guillemin and Sternberg use their canonical form theorem to study certain test complexes associated with a sub-elliptic estimate for (31); we shall use the theorem here to study the test complexes (20) associated with the coercive estimate.

To begin the proof of Theorem 5 let $M \subset M^{\prime}$, let $x \in \partial M$, and let $\zeta_{0}^{\prime} \in T_{x}^{*}(M)$ be orthogonal to $d r$. Then for each $s$ we can form a test complex

$$
C^{\infty} L_{2}\left(\boldsymbol{R}_{+}, E_{x}^{s-1}\right) \stackrel{a\left(\zeta_{0}^{\prime}+d r D_{t}\right)}{\longrightarrow} C^{\infty} L_{2}\left(\boldsymbol{R}_{+}, E_{x}^{s}\right) \stackrel{a\left(\zeta_{0}^{\prime}+d r D_{t}\right)}{\longrightarrow} C^{\infty} L_{2}\left(\boldsymbol{R}_{+}, E^{s+1}\right)
$$

analogous to (19). If the symbol sequence (32) is exact for all $\zeta=\zeta_{0}^{\prime}+\lambda d r$, then Theorem 4 shows that (37) is exact; hence we may assume that $\zeta_{0}=$ $\zeta_{0}^{\prime}+\lambda_{0} d r \in \mathscr{V}$ for some $\lambda_{0} \in C$, and the Guillemin-Sternberg canonical form theorem applies. As Guillemin and Sternberg show, since the complex (31) is elliptic, the neighborhood $U$ can be chosen with $U \supset\left\{\zeta_{0}^{\prime}+\lambda d r \mid \lambda \in C\right\}$, and $R$ 
can be chosen so that its symbol is constant on $\left\{\zeta_{0}^{\prime}+\lambda d r \mid \lambda \in C\right\}$. If $R_{0}$ denotes the constant value of the principal symbol of $R$ on this set, then the homology of (37) remains the same when we replace each of the operators $a\left(\zeta_{0}^{\prime}+d r D_{t}\right)$ by its conjugate $R_{0} a\left(\zeta_{0}^{\prime}+d r D_{t}\right) R_{0}^{-1}$. Now the new complex formed by making these replacements is the direct sum of two complexes, one arising from the symbol sequence of (34) and the other arising from the symbol sequence of (35). Since the symbol sequence of (34) is exact, Theorem 4 shows that the first of these complexes is exact. Hence the homology of (37) is isomorphic to the homology of

$$
\begin{aligned}
& C^{\infty} L_{2}\left(\boldsymbol{R}_{+}, E_{s^{\prime}}^{\prime s_{-1}}\right) \stackrel{a^{\prime \prime}\left(\zeta_{0}^{\prime}+d r D_{t}\right)}{\longrightarrow} C^{\infty} L_{2}\left(\boldsymbol{R}_{+}, E_{x}^{\prime \prime s}\right) \\
& \stackrel{a^{\prime \prime}\left(\zeta_{0}^{\prime}+d r D_{t}\right)}{\longrightarrow} C^{\infty} L_{2}\left(\boldsymbol{R}_{+}, E_{x}^{\prime \prime s+1}\right),
\end{aligned}
$$

where $a^{\prime \prime}(\zeta)$ is the principal symbol of $D^{\prime \prime}$. In view of (36) we have good information about $a^{\prime \prime}(\zeta)$; in fact, it follows from the proof of the canonical form theorem that

$$
a^{\prime \prime}\left(\zeta_{0}^{\prime}+\lambda d r\right) u=\sum_{i=1}^{q} w^{i} \wedge B^{i} u+w^{q} \wedge \lambda u
$$

where the $B^{i}$ 's are commuting linear transformations from $F$ to $F$ which do not depend on $\lambda \in C$, and thus

$$
a^{\prime \prime}\left(\zeta_{0}^{\prime}+d r D_{t}\right)=\sum w^{i} \wedge B^{i} u+w^{q} \wedge D_{t} u .
$$

The vector space $W$ can be decomposed into a direct sum of subspaces which are invariant and indecomposable relative to $B^{1}, \cdots, B^{q}$, and the complex (38) splits correspondingly into direct sum. Thus we may assume that $W$ is already indecomposable for $B^{1}, \cdots, B^{q}$ so that for each $i$ we have

$$
B^{i}=b^{i}+N^{i},
$$

where $b^{i} \in C$, and $N^{i}$ is nilpotent. If any one of $b^{1}, \cdots, b^{q-1}$ is not 0 , then it follows (see the remark following the proof of Lemma 1, below) that the mappings $a^{\prime \prime}\left(\zeta_{0}^{\prime}+\lambda d r\right)$ form an exact sequence for every $\lambda \in C$, and hence by the proof of Theorem 4 the test complex (38) is exact. Thus the proof of Theorem 5 reduces to determining the homology of

$$
\begin{aligned}
C^{\infty} L_{2}\left(\boldsymbol{R}_{+}, \Lambda^{s-1} W \otimes F\right) \stackrel{d}{\longrightarrow} & C^{\infty} L_{2}\left(\boldsymbol{R}_{+}, \Lambda^{s} W \otimes F\right) \\
& \stackrel{d}{\longrightarrow} C^{\infty} L_{2}\left(\boldsymbol{R}_{+}, \Lambda^{s+1} W \otimes F\right),
\end{aligned}
$$

where 


$$
d u=\sum_{i=1}^{q} w^{i} \wedge N^{i} u+w^{q} \wedge\left(D_{t}+b^{q}\right) u
$$

In Lemmas 1 and 2 below we shall show that (39) is exact if $s=q$, but fails to be exact if $0 \leq s<q$ and $\operatorname{Im} b^{q}<0$. Before proving these lemmas let us see that this will complete the proof of Theorem 5. In fact, if (39) is always exact for $s=q$, then so is (37), and by Theorem 2 the coercive estimate holds at $E^{s}$ for all $M \subset M^{\prime}$. Thus by Theorem 3 the complex (31) is locally exact at $E^{q}, \cdots, E^{n}$. Also if ${ }^{\partial} \mathscr{V}_{x} \cap T_{x}^{*}(\partial M)=0$ for each $x \in \partial M$, it follows by Theorem 4 that the coercive estimate holds at each $E^{s}$. On the other hand, if $0 \leq s<q$ and if for some $x \in \partial M$ the characteristic variety $\mathscr{V}_{x}$ contains $\zeta_{0}=$ $\zeta_{0}^{\prime}+\lambda_{0} d r$ with $\lambda_{0} \neq 0$ and $\zeta_{0}^{\prime} \in T_{x}^{*}(\partial M)$, then (37) can be exact only if (39) is exact. But by replacing $\zeta_{0}$ by $-\zeta_{0}$ if necessary we can arrange that $\operatorname{Im} b^{q}<0$ so that (39) is not exact. By Theorem 1 the coercive estimate does not hold at $E^{s}$.

Lemma 1. Let $N^{1}, \cdots, N^{q}$ be commuting nilpotent linear transformations on the vector space $F$, let $w^{1}, \cdots, w^{q}$ form a basis for the vector space $W$, and for each $s \geq 0$ define $\delta: \Lambda^{s} W \otimes F \rightarrow \Lambda^{s+1} W \otimes F$ by $\delta u=\sum_{i=1}^{q} w^{i} \wedge N^{i} u$. Then for $s=0, \cdots, q$ the homology of

$$
\Lambda^{s-1} W \otimes F \stackrel{\delta}{\longrightarrow} \Lambda^{s} W \otimes F \stackrel{\delta}{\longrightarrow} \Lambda^{s+1} W \otimes F
$$

is nonzero.

Proof (by induction on $q$ ). If $q=1$, the lemma holds trivially. To prove the inductive step let $q>1$ and assume the lemma is true when $q$ is replaced by $q-1$. From the filtration

$$
\Lambda \cdot W \otimes F \supset\left\{u \in \Lambda \cdot W \otimes F \mid w^{q} \wedge u=0\right\} \supset 0
$$

arises a spectral sequence $\left\{E_{r}^{p, s}\right\}$ such that in filtration degree zero

$$
E_{0}^{p, 0}=\Lambda^{p} W^{\prime} \otimes F, \quad \delta_{0} u=\sum_{i=1}^{q-1} w^{i} \wedge N^{i} u, \quad u \in E_{0}^{p, 0},
$$

where $W^{\prime}$ is the subspace of $W$ spanned by $w^{1}, \cdots, w^{q-1}$. Also the complex $\left\{E_{0}^{, 1}, \delta_{0}\right\}$ arises from the complex $\left\{E_{0}^{,{ }^{0},}, \delta_{0}\right\}$ by multiplication with $w^{q}$. To compute the homology of (40), therefore, one should compute the homology groups $H^{s-1}$ and $H^{s}$ of the complex $\left\{E^{,}, 0, \delta_{0}\right\}$ at the positions $E_{0}^{s-1,0}$ and $E_{0}^{s, 0}$ and then compute the kernel of the induced map $N^{q}: H^{s} \rightarrow H^{s}$ and the cokernel of $N^{q}: H^{s-1}$ $\rightarrow H^{s-1}$. The homology of (40) is the direct sum of this kernel and cokernel. Now by the inductive hypothesis $H^{s} \neq 0$ for $0 \leq s \leq q-1$, and the induced map $N^{q}: H^{s} \rightarrow H^{s}$ is clearly nilpotent. Hence this map will have nonzero kernel and cokernel for $0 \leq s \leq q-1$, and it follows that the homology of (40) is nonzero when $0 \leq s \leq q$. 
Remark. Note that if $N^{1}, \cdots, N^{q}$ are arbitrary commuting linear transformations one of which, say $N^{q}$, is bijective, then the homology of (40) must vanish. In fact, this is because in the argument above $N^{q}$ induces an isomorphism $N^{q}: H^{s} \rightarrow H^{s}$ for each $s$.

Lemma 2. If $N^{1}, \cdots, N^{q}, F$, and $W$ are as in Lemma 1, then (39) is always exact when $s=q$, but (39) fails to be exact when $0 \leq s<q$ and $\operatorname{Im} b^{q}<0$.

Proof. To compute the homology of (39) we use the spectral sequence corresponding to the filtration

$$
C^{\infty} L_{2}\left(\boldsymbol{R}_{+}, \Lambda \cdot W \otimes F\right) \supset\left\{u \in C^{\infty} L_{2}\left(\boldsymbol{R}_{+}, \Lambda \cdot W \otimes F\right) \mid w^{q} \wedge u=0\right\} \supset 0 .
$$

Then in this spectral sequence

$$
E_{0}^{p, 0}=E_{0}^{p, 1}=C^{\infty} L_{2}\left(\boldsymbol{R}_{+}, \Lambda^{p} W^{\prime} \otimes F\right),
$$

where $W^{\prime}$ is the subspace of $W$ spanned by $w^{1}, \cdots, w^{q-1}$, and the differential in degree zero is the mapping $\delta_{0}$ occurring in the proof of Lemma 1 . It follows that the homology of $\left\{E_{0}^{\cdot, 0}, \delta_{0}\right\}$ at the sth position is $C^{\infty} L_{2}\left(R_{+}, H^{s}\right)$, where $H^{s}$ is the vector space occurring in the proof of Lemma 1 , and hence the homology of (39) is the direct sum of

$$
\operatorname{ker}\left(\left(D_{t}+b^{q}+N^{q}\right): C^{\infty} L_{2}\left(\boldsymbol{R}_{+}, H^{s}\right) \rightarrow C^{\infty} L_{2}\left(\boldsymbol{R}_{+}, H^{s}\right)\right)
$$

and

$$
\operatorname{coker}\left(\left(D_{t}+b^{q}+N^{q}\right): C^{\infty} L_{2}\left(\boldsymbol{R}_{+}, H^{s-1}\right) \rightarrow C^{\infty} L_{2}\left(\boldsymbol{R}_{+}, H^{s-1}\right)\right) .
$$

Now the space (42) is always 0 because if $f \in C^{\infty} L_{2}\left(R_{+}, H^{s-1}\right)$, we can define $f(t)=0$ for $t<0$, and then

$$
u(t)=(2 \pi)^{-1} \int_{-\infty}^{\infty} e^{i\langle t, \tau\rangle}\left(\tau+b^{q}+N^{q}\right)^{-1} \tilde{f}(\tau) d \tau
$$

belongs to $C^{\infty} L_{2}\left(\boldsymbol{R}_{+}, H^{s-1}\right)$ and satisfies $\left(D_{t}+b^{q}+N^{q}\right) u(t)=f(t)$ for $t \geq 0$. Since $H^{q}=0$, it now follows that (39) is exact when $s=q$. However, if $0 \leq s<q$, then by Lemma 1 we know that $H^{s} \neq 0$ and hence the equation $\left(D_{t}+b^{q}+N^{q}\right) u=0$ has a nonzero solution of the form $u(t)=u(0) \exp \left(-i b^{q} t\right)$. Thus (41) will be nonzero if $\operatorname{Im} b^{q}<0$, and the proof of Lemma 2 is complete.

If the characteristic variety $\mathscr{V}=0$, then by Theorem 4 the coercive estimate holds at all positions and for all manifolds $M$. There also exist complexes like (31) for which $\mathscr{V} \neq 0$ and for which the coercive estimate holds at all positions for some choices of $M$. For example, let $\Omega$ be a compact complex manifold of complex dimension $m$, let " $T(\Omega)$ denote its holomorphic tangent bundle, and let $M=\Omega \times I$. Define

$$
E^{s}=\Lambda^{s}\left(\overline{(\prime T(\Omega)} * T(I)^{*}\right)
$$


for $0 \leq s \leq m+1$, and let $\mathscr{D}: E^{s} \rightarrow E^{s+1}$ be the differential operator defined in terms of local coordinates by

$$
\mathscr{D} u=\sum_{j=1}^{m} d \bar{z}^{j} \wedge \frac{\partial}{\partial \bar{z}^{j}} u(z, t)+d t \wedge \frac{\partial}{\partial t} u(z, t) .
$$

Then these operators form a differential complex of length $m+1$, and $\mathscr{V}_{(z, t)}$ $={ }^{\prime \prime} T_{z}(\Omega) *$ for all $(z, t) \in M$. Hence $q=m+1$, all of the characteristics are Cohen-Macaulay. Since ${ }^{\partial \mathscr{V}}(z, t)={ }^{\prime \prime} T_{z}(\Omega)^{*}$, when $t=0$, 1 , Theorem 5 shows that the coercive estimate holds at all positions. In [1] M. E. Ash obtained this result by direct computation.

In spite of the example just given one should expect the coercive estimate to fail at the positions $E^{0}, \cdots, E^{q-1}$ when $\mathscr{V} \neq 0$. In fact, given any $x \in M^{\prime}$ we can then choose a nonzero element $\zeta$ of $\mathscr{V}_{x}$, and the estimate must fail at these positions for any manifold $M$ with $T(\partial M)$ orthogonal to $\operatorname{Im} \zeta$. Also, in the case of a complex of constant-coefficient operators over $M \subset \boldsymbol{R}^{n}$, if $\zeta$ is any nonzero element of $\mathscr{V}_{x}$ and if $M \subset M^{\prime}$ is any compact submanifold, then we must have $\operatorname{Im} \zeta=d r$ for at least one point in $\partial M$, and hence the coercive estimate fails for sections of $E^{0}, \cdots, E^{q-1}$ over $M$.

Added in proof. Another treatment of some of the work in [4] will appear in the Princeton $\mathrm{Ph}$. D. thesis of Charles Rockland, who also points out that, in general, (34) is a complex only modulo terms of order 0 . A report by D. C. Spencer on this work will be contained in the Proceedings of the Amer. Math. Soc. 1971 Summer Conference.

\section{References}

[1] M. E. Ash, The Neumann problem on strongly pseudoconvex complex multifoliate manifolds, Princeton University thesis, 1962, unpublished.

[2] D. G. De Figueiredo, The coerciveness problem for forms over vector valued functions, Comm. Pure Appl. Math. 16 (1963) 63-94.

[ 3 ] V. W. Guillemin, D. G. Quillen \& S. Sternberg, The integrability of characteristics, Comm. Pure Appl. Math. 23 (1970) 39-77.

[4] V. W. Quillemin \& S. Sternberg, Subelliptic estimates for complexes, Proc. Nat. Acad. Sci. U.S.A. 67 (1970) 271-274.

[5] L. Hörmander, An introduction to complex analysis in several variable, Van Nostrand, Princeton, New Jersey, 1966.

[6] —, Linear partial differential operators, Springer, Berlin, 1963.

[7] Y. Kato, The Poisson kernels and the Cauchy problem for elliptic equations with analytic coefficients, Trans. Amer. Math. Soc. 144 (1969) 321-331.

[8] C. B. Morrey, Jr. \& L. Nirenberg, On the analyticity of solutions of linear elliptic systems of partial differential equations, Comm. Pure Appl. Math. 10 (1957) 271-290.

[9] D. C. Spencer, Overdetermined systems of linear partial differential equations, Bull. Amer. Math. Soc. 75 (1969) 179-239.

[10] W. J. Sweeney, The D-Neumann problem, Acta Math. 120 (1968) 223-277.

PRINCETON UNIVERSITY RUTGERS UNIVERSITY 
